infections and development of type 1 diabetes: an evasive conundrum. J. Pediatr. Gastroenterol. Nutr. 45:147-156

16. Surh, C.D., Boyman, O., Purton, J.F., and Sprent, J. 2006. Homeostasis of memory T cells. Immunol. Rev. 211:154-163.

17. Dooms, H., and Abbas, A.K. 2006. Control of CD4+ T-cell memory by cytokines and costimulators. Immunol. Rev. 211:23-38.

18. Purton, J.F., et al. 2007. Antiviral CD4+ memory T cells are IL-15 dependent. J. Exp. Med. 204:951-961.

19. de Kleer, I., et al. 2006. Autologous stem cell transplantation for autoimmunity induces immunologic self-tolerance by reprogramming autoreactive $\mathrm{T}$ cells and restoring the CD4+CD25+ immune regulatory network. Blood. 107:1696-1702.
20. Malek, T.R., and Bayer, A.L. 2004. Tolerance, not immunity, crucially depends on IL-2. Nat. Rev. Immunol. 4:665-674.

21. Boyman, O., Kovar, M., Rubinstein, M.P., Surh, C.D., and Sprent, J. 2006. Selective stimulation of $\mathrm{T}$ cell subsets with antibody-cytokine immune complexes. Science. 311:1924-1927.

22. NIDDK. New onset of type 1 diabetes mycophenolate mofetil-daclizumab clinical trial [ClinicalTrials. gov study NCT00100178]. http://www.clinicaltrials. gov/ct2/show/NCT00100178.

23. Keymeulen, B., et al. 2005. Insulin needs after CD3antibody therapy in new-onset type 1 diabetes. N. Engl. J. Med. 352:2598-2608.

24. Bresson, D., et al. 2006. Anti-CD3 and nasal proinsulin combination therapy enhances remission from recent-onset autoimmune diabetes by inducing Tregs. J. Clin. Invest. 116:1371-1381.

25. NIAID. Islet transplantation in type 1 diabetes [ClinicalTrials.gov study NCT00434811]. http:// www.clinicaltrials.gov/ct2/show/NCT00434811.

26. Nir, T., Melton, D.A., and Dor, Y. 2007. Recovery from diabetes in mice by beta cell regeneration. J. Clin. Invest. 117:2553-2561.

27. Bayhill Therapeutics. Phase 1 study of BHT-3021 in subjects with type 1 diabetes mellitus [ClinicalTrials.gov study NCT00453375]. http://www.clinicaltrials.gov/ct2/show/NCT00453375.

28. NIDDK. Effects of recombinant human glutamic acid decarboxylase [ClinicalTrials.gov study NCT00529399]. http://www.clinicaltrials.gov/ct2/ show/NCT00529399.

\title{
What lurks beneath: IL-11, via Stat3, promotes inflammation-associated gastric tumorigenesis
}

\author{
Juanita L. Merchant \\ Department of Internal Medicine and Department of Molecular and Integrative Physiology, \\ Division of Gastroenterology, University of Michigan, Ann Arbor, Michigan, USA.
}

\begin{abstract}
Chronic inflammation in the stomach induces cellular transformation and gastric cancer primarily in the distal stomach or antrum. In this issue of the JCI, a study in mice by Ernst et al. provides new insight into the role of IL-11 and its glycoprotein 130 (gp130) receptor in inflammation-associated gastric epithelial cell oncogenic transformation, which they show is mediated by and dependent on increased activation of Stat 3 and, to a lesser extent, Stat1 (see the related article beginning on page 1727). Prior studies from this group have shown that Stat 3 hyperactivity stimulates the TGF- $\beta$ inhibitor Smad7. Collectively, the studies suggest that an important pathway of oncogenic transformation in the stomach is through suppression of growth inhibitory signals, such as members of the TGF- $\beta$ family, that originate from the stroma.
\end{abstract}

Cancer originates in a variety of different tissues that are susceptible to chronic inflammatory conditions. In the stomach, the phenotypic steps leading from chronic inflammation (gastritis) to distal gastric cancer were established epidemiologically (1) before the discovery of Helicobacter pylori, a noninvasive curvilinear rod that resides in the luminal mucous layer (2). Although H. pylori appears to be the most common infectious agent inducing chronic gastritis, studies support the notion that inflammatory cells infiltrate the mucosa from the stroma beneath and secrete cytokines that are ostensibly the direct modulators of epithelial cell differentiation and eventually neoplastic transformation $(3,4)$. If $H$. pylori is not eradi-

Nonstandard abbreviations used: gp130, glycoprotein 130; Smad7, mothers against decapentaplegic homolog 7 .

Conflict of interest: The author has declared that no conflict of interest exists.

Citation for this article: J. Clin. Invest. 118:1628-1631 (2008). doi:10.1172/JCI35344. cated by the inflammatory response, the bacterium becomes a chronic trigger for the immune system to produce cytokines that continue to bathe the gastric glands. Metaplastic changes begin in the antrum, where H. pylori preferentially colonizes the stomach, before spreading anteriorly into the corpus, inducing atrophy of the oxyntic glands (5). Eventually, mucus-producing, metaplastic cells that exhibit features of either the antral pyloric glands (via antralization) or small intestine replace the normal oxyntic (acid/ pepsin-producing) cells $(6,7)$. These specific changes in the gastric lineages launch the mucosa down an irreversible pathway toward unregulated growth, dysplasia, and eventually neoplastic transformation.

Population-based studies by El-Omar et al. indicate that IL- $1 \beta$ is one of the essential proinflammatory cytokines modulated during H. pylori infection that directs the mucosa toward atrophy, metaplasia, and neoplastic transformation (8). In European, Asian, and Hispanic populations, the presence of a gas- tric cancer-associated polymorphism has not only been confirmed for IL- $1 \beta$, but also reported for TNF- $\alpha$, IL-10, and IL-6 (9-11). Collectively, these prior studies have set in motion the quest to link inflammation and specific cytokines with cancer development. The underlying issue is that once inflammation becomes chronic, antibiotic eradication of the triggering organism would not be an effective treatment. It remains to be seen whether antiinflammatory therapies that do not disrupt mucosal restitution can be used.

\section{A fully penetrant model of distal gastric cancer}

To study the etiology of gastric transformation, a number of mouse models have been developed that recapitulate several features observed in human intestinal-type gastric cancer (Table 1). The gp1307757F/Y757F mouse studied by Ernst et al. in this issue of the JCI (12) is the mouse model that most consistently develops gastric cancer. These animals are homozygous for a phenylalanine knockin substitution at Tyr757, which is present in one of five major phosphorylation sites in the cytoplasmic tail of the glycoprotein 130 (gp130) receptor. This receptor - the common receptor for members of the IL- 6 cytokine family - is important for signal transduction following cytokine engagement. Upon binding of an IL-6 cytokine family member to its relevant receptor, the complex of these two proteins associates with gp130 and homodimerizes to form a hexameric complex that upon phosphorylation 


\section{Table 1}

Mouse models recapitulating features of human intestinal-type gastric cancer

\begin{tabular}{|c|c|c|c|c|}
\hline Mouse model & Site of tumor & Corpus atrophy & Signaling pathway & Reference \\
\hline gp130 Y757F/Y757F & Antrum & No & STAT & 14 \\
\hline Tff1-null & Antrum & No & ERK & 24 \\
\hline Runx3-null & Antrum & Unknown & TGF- $\beta$ & 25 \\
\hline Smad4++- plus LOH & Corpus & Yes & TGF- $\beta$ & 26 \\
\hline Gastrin-null & Antrum & Yes & Unknown & 27 \\
\hline INS-GAS with Helicobacter felis infection & Corpus & Yes & Unknown & 28 \\
\hline H. pylori infection in Mongolian gerbils & Antrum & Yes & $\beta$-catenin & 29 \\
\hline Elf+/ (a $\beta$-spectrin $)$ & Antrum & No & TGF- $\beta$ & 30 \\
\hline Cdx2 overexpression & Antrum or corpus & Yes & Unknown & 31,32 \\
\hline$p 53^{+/-}$plus MNU & Antrum & No & Unknown & 33 \\
\hline$T G F-\beta^{+/-}$ & Antrum & Unknown & TGF- $\beta$ & 34 \\
\hline
\end{tabular}

ELF, embryonic liver fodrin; INS-GAS, insulin-gastrin transgenic mice; LOH, loss of heterozygosity; MNU, $N$-methyl- $N$-nitrosourea.

can activate the ERK signaling kinase and STAT protein transcription factors, leading to the activation of downstream genes.

The time course of cancer development in the gp130 Y757F/Y757F animals is rapid and fully penetrant, in comparison to that of gastric cancer in humans, which emerges over decades in about $1 \%$ of chronically $H$. pyloriinfected subjects (13). This observation suggests that there are likely several pathways parallel to or converging upon the genetic targets downstream of the gp130 receptor. Also, since these mice do not develop the well-recognized preneoplastic lesions that typically emerge with chronic gastritis (e.g., corpus atrophy or mucous cell metaplasia) these precursor events cannot be investigated in this model. Despite such differences between this animal model and the human disease, Ernst et al. (12) analyzed the rapidly evolving antral cancers in these mutant mice in order to provide novel mechanistic insights into the events immediately proximal to neoplastic transformation.

\section{IL-11, not IL-6, activates gp130- mediated tumorigenesis}

There are several ligands known to activate the gp130 receptor, with IL-6 arguably being the best known of the cytokine family members linked to gastric transformation (14). By contrast, little is known regarding the role of another gp130 ligand, IL-11, in gastric transformation, and IL-11 has only recently been examined in the setting of human gastric cancers (15). A recent study by Ernst and colleagues revealed that megakaryocyte development also depends upon gp130 activation of STAT3 (16). In that study, gp130 in bone marrow progenitors appeared to be activated by IL- 6 , rather than IL- 11 as described in the current report (12) for the stomach.
Thus, the various ligands appear to impart to gp130 its tissue-specific activation. For example, IL-11 activation of the gp130 receptor is pro-oncogenic in the stomach but not in bone marrow-derived cells. In fact, a few studies have implicated IL-11 in gastric restitution through proangiogenic and proproliferative mechanisms $(17,18)$. Nevertheless, the regulators of IL-11 expression during gastric inflammation are unknown. Consequently, the production of IL-11 during $H$. pylori infection will need to come under greater scrutiny in future studies of chronic gastritis, atrophy, and cancer.

In their current study reported in this issue of the JCI, Ernst et al. (12) showed that it is indeed IL-11 that is responsible for the aberrant activation of this mutant receptor in gastric tumorigenesis, using an elegant display of mouse genetics in which the gp130 Y757F/Y757F mutation was bred onto either an IL-6- or IL-11 $\alpha$ receptor-null background. As noted above, the normal gp130 receptor is able to activate both the ERK and STAT3 pathways $(14,19)$. The Y757F point mutation in the gp130 Y757F/Y757F mouse abrogates phosphorylation at that residue and prevents the receptor from docking with the SRC homology 2 domain-containing (SH2-containing) tyrosine phosphatase (Shp2) and thereby initiating signal transduction via the Ras/Erk or PI3K/Akt pathways (Figure 1). This in turn allows exclusive receptor activation of the Stat1/Stat3 pathway to proceed via the other four C-terminal receptor tyrosines upon ligand binding. Prior deletion and mutational analysis of various combinations of these five $\mathrm{C}$-terminal tyrosines revealed that gp130 receptor-mediated activation of the Erk pathway is proapoptotic and blocks the immune response, whereas activation of the Stat3 pathway is proproliferative and ultimately oncogenic (19).

In addition to identifying the preferred ligand activating the mutant gp130 receptor, Ernst et al. (12) used antisense oligonucleotides to further discern whether the downstream effects of this activation were preferentially mediated by Stat 3 or Stat 1 . Moreover, these experiments were designed to test whether the antisense oligonucleotides were effective therapy to prevent tumor development after systemic administration. Indeed, both Stat 1 and Stat 3 antisense oligonucleotides abrogated tumor development in these animals, although the effect was greater using the Stat 3 antisense oligonucleotides. The ability to reverse or block tumor growth initiated by cytokine receptor activation is quite exciting, since the result underscores that the cascade of events leading to tumor formation might still be reversible. However, most clinicians would view the latter statement cautiously, given the abysmal results observed with gastric cancer therapies in human subjects (20). Generally, human epithelial cancer cells recruit multiple signaling pathways and mechanisms to survive, thereby circumventing current therapies. Thus, complete destruction of gastric cancers is expected to require the identification and targeting of multiple pathways, many of which currently remain undiscovered, in order for combination therapies to be effectively designed.

\section{Activated Stat3 induces inhibitory Smad7}

Relevant to the mechanism underlying Stat3 regulation is the observation that Stat 3 participates in crosstalk between the mesenchyme and epithelium $(21,22)$. In a prior study by Ernst and colleagues, hyperactive Stat 3 signaling induced expression of the 


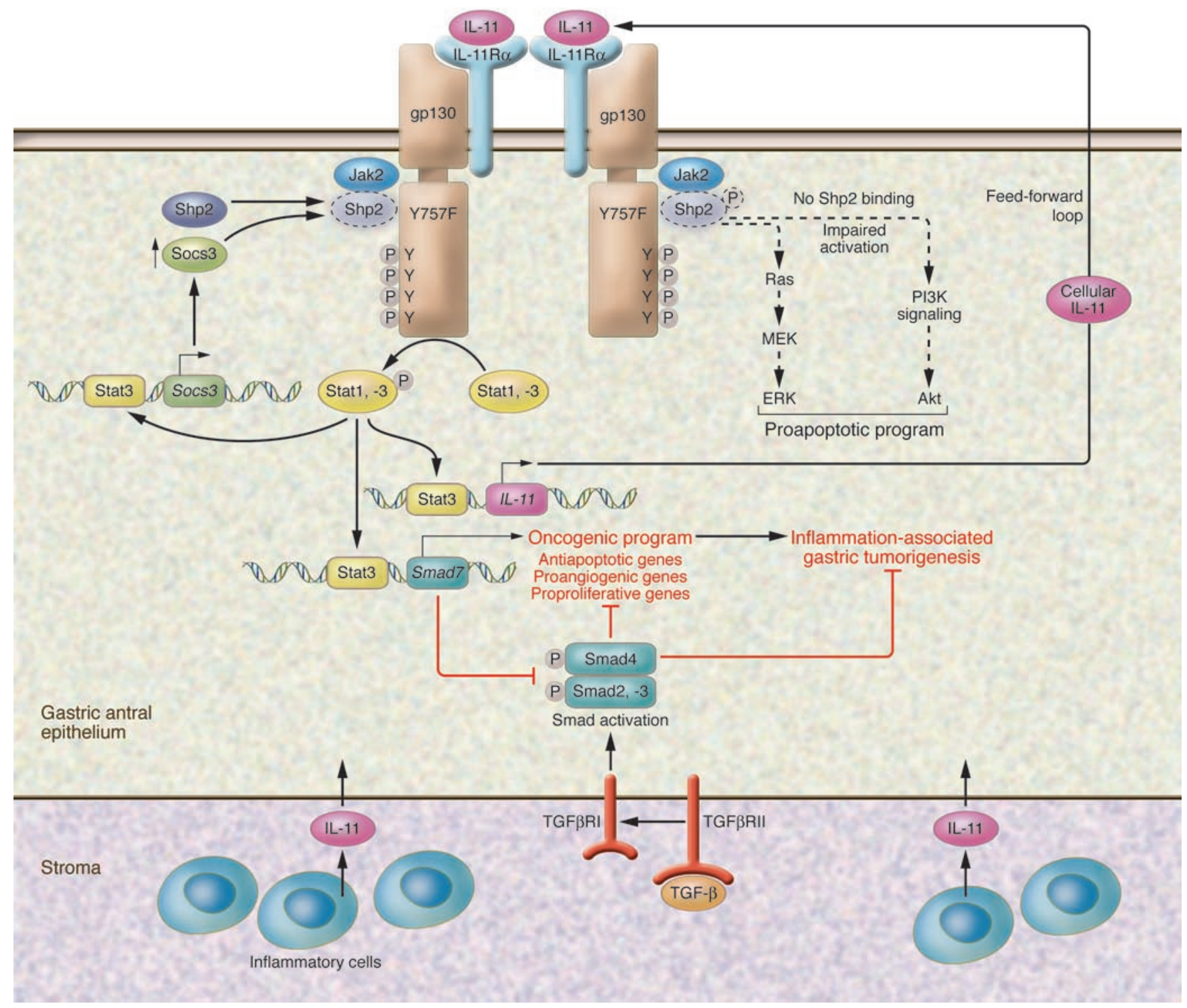

Figure 1

Mechanism of IL-11-mediated induction of gp130 and gastric tumorigenesis. Schematic of mucosal changes that occur during chronic gastric inflammation, in which IL-11 is one of the cytokines produced. IL-11 binds to the IL-11 receptor $\alpha$ (IL-11R $\alpha$ ) subunit that heterodimerizes with the gp130 homodimeric receptor. IL-11 is presumably produced by both the epithelium and inflammatory cells and binds to the gp130 receptor also located on epithelial cells in the gastric antrum. As described in the current study by Ernst et al. (12), in the presence of the Y757F mutation in the gp130 receptor, $\mathrm{SRC}$ homology 2 domain-containing (SH2-containing) tyrosine phosphatase (Shp2) phosphorylation and subsequent activation of the proapoptotic Ras/Erk and PI3K/AKT pathways do not occur. Instead, phosphorylation of the four C-terminal tyrosine residues in the gp130 receptor, in the absence of phosphorylation of Tyr757, results in Stat activation. Stat3 is activated to a greater extent than Stat1. Suppressor of cytokine signaling 3 (Socs3) is a downstream target of Stat3 and competes with Shp2 for docking at Tyr757. Therefore, epithelial Socs3 levels in the cells rise, but they cannot bind gp130 receptor and prevent its signaling. In addition, Stat activation induces the expression of the inhibitory protein Smad7, which blocks TGF- $\beta$-activated Smad signaling. In this way, Stat3 hyperactivity induced by the IL-11 proinflammatory cytokine can suppress the cytostatic effect of the stroma on cell proliferation (22). Moreover, Stat3 also induces epithelial cell expression of IL-11, setting a feed-forward mechanism that fuels persistent cellular proliferation. Collectively, these signaling events promote an oncogenic program in which the expression of antiapoptotic, proangiogenic, and proproliferative genes results in inflammation-associated gastric tumorigenesis. F, phenylalanine; P, phosphate; TGF $\beta R$, TGF- $\beta$ receptor; Y, tyrosine.

inhibitory mothers against decapentaplegic homolog 7 (Smad7) protein, which blocks the cytostatic effect of TGF- $\beta$ signaling (22). This study (22) underscores the existence of crosstalk between Stat 3 signaling in the epithelium and TGF- $\beta$ regulation from the stroma. The authors do not describe which cell types express IL-11 and perhaps more importantly which cells express the gp130 receptor (12). However, one would presume that the receptor is located on the basolateral membrane of responding epithelial cells, as has been reported for other polarized epithelial (23) and ostensibly tumor cells.

Given the putative link between overactive Stat 3 signaling and the Smad pathway in the epithelium, one might envision that the hyperplastic cells produce an inhibitor that 
limits the growth-restrictive influence of the stroma or mesenchyme. For example, hyperactive Stat 3 induces Smad7 expression, then Smad7 blocks activator Smads (Smad2, -3 , -4) that normally transduce TGF- $\beta$ signals originating from the mesenchyme (Figure 1). Mesenchymal cells present in the basement membrane and lamina propria form the support structure for the gastric epithelium and are the major source of TGF- $\beta$ family members (TGF- $\beta$, activins, inhibins, and bone morphogenetic proteins). As in other epithelia, there is a synergistic relationship between the gastric mesenchymal and epithelial compartments that instructs the endoderm during development, then restricts uncontrolled growth by balancing cell death and cell growth signals once the mucosa has matured.

In addition to the stroma, some inflammatory cells also express TGF- $\beta$. However, Ernst et al. (12) anticipated this possibility and used adoptive transfer of bone marrowderived cells expressing the gp130 Y757F/Y757F mutation into irradiated mice to prove that the tumor development was not related to hyperactive signaling from the inflammatory cells. Interestingly, several of the mouse models of gastric cancer also represent perturbations in the TGF- $\beta$ pathway (Table 1 ), supporting the contention that the negative influences exerted by the stroma are required to keep the epithelium in check. Since most of the tumors that arise in the antrum correlate with disruptions of the TGF- $\beta$ pathway, one might also query why the antral epithelium is more susceptible to hyperactivation of this signaling pathway.

There are no known mutations or changes in methylation status of the endogenous gp130 receptor locus. Moreover, if mutations did occur, they would likely result in the occurrence of tumors more frequently and at an earlier age. Although not observed here in the US, there might be populations in which the gp130 locus is modified, especially those with a high prevalence of $H$. pylori infection and with an increased susceptibility to gastric cancer.

In summary, the current study by Ernst et al. (12) reinforces the critical importance of the IL-11/Stat3 signal transduction pathway in gastric tumors in mice, as well as the importance of TGF- $\beta$ signaling in restricting the hyperproliferation of the epithelium. Future studies should determine whether these pathways are disrupted in susceptible patient populations and examine how they are regulated by chronic inflammation. The $g p 130^{Y 757 F / Y 757 F}$ receptor mouse model will be useful for sorting out why the distal stomach (lesser curvature and pyloric [antral] glands) is more susceptible to hyperplastic Stat 1 and Stat 3 signaling compared with the corpus. By contrast, other disease models that exhibit a more protracted course to transformation will need to be examined to provide insight into the relationship between precursor lesions (atrophy and mucous gland metaplasia) and the irreversible progression to cancer.

\section{Acknowledgments}

This work was supported in part by Public Health Service Grant R01-DK61410 (to J.L. Merchant).

Address correspondence to: Juanita L. Merchant, Departments of Internal Medicine and Molecular and Integrative Physiology, University of Michigan, 109 Zina Pitcher Place, BSRB Room 2051, Ann Arbor, Michigan 48198-2200, USA. Phone: (734) 647-2944; Fax: (734) 763-4686; E-mail: merchanj@ umich.edu.

1. Correa, P., Haenszel, W., Cuello, C., Tannenbaum, S., and Archer, M. 1975. A model for gastric cancer epidemiology. Lancet. 2:58-60.

2. Marshall, B.J., and Warren, J.R. 1984. Unidentified curved bacilli in the stomach of patients with gastritis and peptic ulceration. Lancet. 1:1311-1315.

3. Bromberg, J. 2002. Stat proteins and oncogenesis. J. Clin. Invest. 109:1139-1142.

4. Judd, L.M., et al. 2006. STAT3 activation regulates growth, inflammation, and vascularization in a mouse model of gastric tumorigenesis. Gastroenterology. 131:1073-1085.

5. El-Zimaity, H.M., et al. 2002. Patterns of gastric atrophy in intestinal type gastric carcinoma. Cancer. 94:1428-1436.

6. Matsukura, N., et al. 1980. Distribution of marker enzymes and mucin in intestinal metaplasia in human stomach and relation to complete and incomplete types of intestinal metaplasia to minute gastric carcinomas. J. Natl. Cancer Inst. 65:231-240.

7. Tsukamoto, T., Mizoshita, T., and Tatematsu, M. 2006. Gastric-and-intestinal mixed-type intestinal metaplasia: aberrant expression of transcription factors and stem cell intestinalization. Gastric Cancer. 9:156-166.

8. El-Omar, E.M., et al. 2000. Interleukin-1 polymorphisms associated with increased risk of gastric cancer. Nature. 404:398-402.

9. Perez-Perez, G.I., Garza-Gonzalez, E., Portal, C., and Olivares, A.Z. 2005. Role of cytokine polymorphisms in the risk of distal gastric cancer development. Cancer Epidemiol. Biomarkers Prev. 14:1869-1873.

10. Sugimoto, M., et al. 2007. Effects of interleukin-10 gene polymorphism on the development of gastric cancer and peptic ulcer in Japanese subjects. J. Gastroenterol. Hepatol. 22:1443-1449.

11. Gatti, L.L., et al. 2007. Interleukin-6 polymorphisms, Helicobacter pylori infection in adult Brazilian patients with chronic gastritis and gastric adenocarcinoma. Arch. Med. Res. 38:551-555.

12. Ernst, M., et al. 2008. STAT3 and STAT1 mediate IL-11-dependent and inflammation-associated gastric tumorigenesis in gp130 receptor mutant mice. J. Clin. Invest. 118:1727-1738.

13. Asghar, R.J., and Parsonnet, J. 2001. Helicobacter pylori and risk for gastric adenocarcinoma. Semin.
Gastrointest. Dis. 12:203-208.

14. Judd, L.M., et al. 2004. Gastric cancer development in mice lacking the SHP2 binding site on the IL- 6 family co-receptor gp130. Gastroenterology. 126:196-207.

15. Nakayama, T., et al. 2007. Expression of interleukin-11 (IL-11) and IL-11 receptor alpha in human gastric carcinoma and IL-11 upregulates the invasive activity of human gastric carcinoma cells. Int. J. Oncol. 30:825-833.

16. Jenkins, B.J., et al. 2007. Pathologic consequences of STAT3 hyperactivation by IL-6 and IL-11 during hematopoiesis and lymphopoiesis. Blood. 109:2380-2388.

17. Wen, C.Y., et al. 2003. IL-11 up-regulates Tie-2 expression during the healing of gastric ulcers in rats. World J. Gastroenterol. 9:788-790.

18. Wen, C.Y., et al. 2002. Mechanism of the antiulcerogenic effect of IL-11 on acetic acid-induced gastric ulcer in rats. Life Sci. 70:2997-3005.

19. Tebbutt, N.C., et al. 2002. Reciprocal regulation of gastrointestinal homeostasis by SHP 2 and STATmediated trefoil gene activation in gp 130 mutant mice. Nat. Med. 8:1089-1097.

20. Field, K., Michael, M., and Leong, T. 2008. Locally advanced and metastatic gastric cancer: current management and new treatment developments. Drugs. 68:299-317.

21. Yang, Y., et al. 2006. Regulation of transforming growth factor-beta 1 -induced apoptosis and epithelial-to-mesenchymal transition by protein kinase A and signal transducers and activators of transcription 3. Cancer Res. 66:8617-8624.

22. Jenkins, B.J., et al. 2005. Hyperactivation of Stat3 in gp130 mutant mice promotes gastric hyperproliferation and desensitizes TGF-beta signaling. Nat. Med. 11:845-852.

23. Doumanov, J.A., Daubrawa, M., Unden, H., and Graeve, L. 2006. Identification of a basolateral sorting signal within the cytoplasmic domain of the interleukin-6 signal transducer gp130. Cell Signal. 18:1140-1146.

24. Lefebvre, O., et al. 1996. Gastric mucosa abnormalities and tumorigenesis in mice lacking the pS2 trefoil protein. Science. 274:259-262.

25. Li, Q.L., et al. 2002. Causal relationship between the loss of RUNX3 expression and gastric cancer. Cell. 109:113-124.

26. Takaku, K., et al. 1998. Intestinal tumorigenesis in compound mutant mice of both Dpc4 (Smad4) and Apc genes. Cell. 92:645-656.

27. Zavros, Y., et al. 2005. Chronic gastritis in the hypochlorhydric gastrin-deficient mouse progresses to adenocarcinoma. Oncogene. 24:2354-2366.

28. Wang, T.C., et al. 2000. Synergistic interaction between hypergastrinemia and Helicobacter infection in a mouse model of gastric cancer. Gastroenterology. 118:36-47.

29. Franco, A.T., et al. 2005. Activation of beta-catenin by carcinogenic Helicobacter pylori. Proc. Natl. Acad. Sci.U.S. A. 102:10646-10651.

30. Katuri, V., et al. 2006. Critical interactions between TGF-beta signaling/ELF, and E-cadherin/betacatenin mediated tumor suppression. Oncogene. 25:1871-1886.

31. Silberg, D.G., et al. 2002. Cdx2 ectopic expression induces gastric intestinal metaplasia in transgenic mice. Gastroenterology. 122:689-696.

32. Mutoh, H., et al. 2004. Development of gastric carcinoma from intestinal metaplasia in Cdx2-transgenic mice. Cancer Res. 64:7740-7747.

33. Yamamoto, M., et al. 2000. p53 knockout mice (-/-) are more susceptible than $(+/-)$ or $(+/+)$ mice to $\mathrm{N}$-methyl-N-nitrosourea stomach carcinogenesis. Carcinogenesis. 21:1891-1897.

34. Boivin, G.P., Molina, J.R., Ormsby, I., Stemmermann, G., and Doetschman, T. 1996. Gastric lesions in transforming growth factor beta-1 heterozygous mice. Lab. Invest. 74:513-518. 